\title{
Study on the Performance of a Thermo-Electric Generation Model with Two Different Materials of Heat Pipe-Heat Sink
}

\author{
Ali Elghool ${ }^{1,2, *}$, Firdaus Basrawi ${ }^{1}$, Hassan Ibrahim ${ }^{1}$, Thamir K Ibrahim ${ }^{3}$, Shaharin A. \\ Sulaiman $^{4}$, and $M$. Ishak ${ }^{1}$ \\ ${ }^{1}$ Energy Sustainability Focus Group, Faculty of Mechanical Engineering, Universiti Malaysia Pahang \\ 26600 Pekan, Pahang, Malaysia. \\ ${ }^{2}$ Higher Institute of Sciences and Technology, Gharian, Libya. \\ ${ }^{3}$ College of Applied Engineering, Tikrit University, Iraq \\ ${ }^{4}$ Department of Mechanical Engineering, Universiti Teknologi PETRONAS, Bandar Seri Iskandar, \\ 31750 Tronoh, Perak, Malaysia
}

\begin{abstract}
Heat sink lack of design is one reason that negatively affects the performance of thermoelectric modules. As compared to conventional cooling systems equipped with thermoelectric generators (TEG), heat pipe heat sink has various points of interest. Heat pipe heat sink is the most appropriate heat exchanger for medium temperature range under $300{ }^{\circ} \mathrm{C}$. This paper demonstrates the effect of different materials of heat pipe-heat sink on the TEG performance. Two types of heat sinks were tested with TEG, one made from copper while the other from aluminium. The aim is to improve power output of TEG by an appropriate material of fins and metal block with heat pipes. The prototype was experimentally tested and the TEG cold side temperature, voltage and current were measured in both conditions, natural and forced convection. It was found that highest power output was achieved using copper heat sink in the case of forced convection, being 7.7 $\mathrm{W}$ whereas, lowest power output was obtained using aluminium heat sink in the case of natural convection, being $2.67 \mathrm{~W}$. It is evident that copper heat sink is more effective than aluminium heat sink in terms of power output. However, both types of heat sink needs optimisation in terms of power output, cost and economic efficiency, while the results shown in this paper are just in terms of power output.
\end{abstract}

\section{Introduction}

A large part of the energy from oil derivatives is wasted in the process of transformation and utilisation including thermal power, automotive combustions, power plants and residential heating. Previous studies reported that about $66 \%$ of energy used in oil-consuming sectors is lost as wasted heat[1]. Hence, there is an urgent need for technology to recover the waste heat and to influence a more productive use of power to accomplish both energy and environmental security. Thermo-electric generator (TEG), which directly produces electricity from waste heat sources demonstrates promising outcomes in making vital

\footnotetext{
*Corresponding author: mfirdausb@ump.edu.my
} 
assistance to lessening fossil fuel depletion and $\mathrm{CO} 2$ emissions, no matter how small its contribution would be. The Thermoelectric Generator (TEG) is sold state device consists of number of legs (thermocouples). These legs are connected electrically in series and thermally in parallel; they are used to convert heat to electricity through the Seebeck effect. Points of interest of a TEG module incorporate; absence of moving parts, environmental safety, and silent operation. This generator can likewise be controlled in a consistent and exact manner. Researchers have demonstrated expanded enthusiasm utilising thermo-electric innovation in enhancing waste recovery efficiency in the course of three decades[2]. As per Web of Science database, yearly publications in thermoelectric innovation had expanded over the last 15 years from 500 to as high as $2000[3]$.

However, low efficiency in converting heat into electricity has continued to be the main challenge facing the thermal power generator[4]. TEG heat exchanger's (heat sink) lack of design is one reason that causes weakness in the conversion efficiency of the TEG $[5,6]$. These days, there are intensive research in heat sinks with heat pipes that improve heat sinks considerably, with a small temperature drop, because of the use of the latent heat of an internal fluid. Heat pipe heat sink (HPHS) is the most appropriate heat exchanger for the medium temperature range under $300^{\circ} \mathrm{C}$ with TEG [Energy Efficiency Guide for Industry in Asia] which is this paper's work scope. From literature study there have been many research conducted on the TEG, using waste heat as a heat source and finned heat pipes to cool the TEG cold side.

Araiz. M [7] had studied thermo-syphons with phase change in thermo-electric generators in a waste heat recovery application. The thermo-syphons was coil on the hot side as evaporator and another coil on the top working as a condenser to cool down the refrigerant fluid employed (R-134a). Researcher stating that including thermo-syphons on the cold side of the generators improved the net thermo-electric production by $36 \%$ as compared to that obtained with finned dissipaters under forced convection. Song Lv [8] conducted an experimental investigation of three ordinary heat sinks (aluminium finned heat sink, water block, aluminium finned heat pipes) with TEGs. The outcomes demonstrated that the finned heat sink is the most ordinary heat exchanger on account of its effortlessness and low-cost features. In any case, it has a moderately poor performance with the most elevated heat exchanger costing $\$ 15.12 /(\mathrm{W} / \mathrm{K})$. The water heat exchanger has a superior performance due to the better convective coefficient that water has compared to air. In any case, it presents higher utilisation and application, though with low net efficiency. Practically, heat pipe heat exchanger brings down supplementary equipment consumption and offering heat exchanger cost of $\$ 10.67 /(\mathrm{W} / \mathrm{K})$.

In spite of the fact that intensive research have been done in this area, the majority of these studies remain inadequate for ignoring to use cooper fins with heat pipes instead of aluminium fins to study the performance of heat pipe-heat sink. Copper has a more significant effect on the TEG power output. Thus, to address this gap, this paper presents an investigative and analytical comparison between cooper heat pipe-heat sink and aluminium heat pipe-heat sink in terms of TEG power output.

\section{Methodology}

\subsection{Description of the proposed system}

Figures 1 and 2 show the design of the TEG model - one TEG was used in this experiment whereas copper and aluminium heat sinks were used to dissipate heat from the TEG cold side. The TEG module used was $2411 \mathrm{G}-7 \mathrm{~L} 31-15 \mathrm{CX} 1$ manufactured by Custom Thermoelectric (USA) with dimensions of $56 \mathrm{~mm} \times 56 \mathrm{~mm} \times 4.45 \mathrm{~mm}$. The maximum hot 
side temperature and cold side temperature was $320^{\circ} \mathrm{C}$ and $180^{\circ} \mathrm{C}$, respectively. The TEG hot side was located on a heater to simulate the source of waste heat. Heat moved from the hot side to the cold side of the TEG. The cold side was attached to the metallic block (copper or aluminium) where five heat pipes of $50 \mathrm{~W}$ each were inserted in it. On the other side of the heat pipes were fins to eject heat to the surroundings. There were twenty seven aluminium fins (A1100) bounded mechanically on the heat pipes, and similar for the copper heat sink. All copper and aluminium fins have a rectangular size of $118 \times 70 \times 0.5 \mathrm{~mm}$, with $3 \mathrm{~mm}$ fin space. Five pieces of copper heat pipes with $200 \mathrm{~mm}$ long and $8 \mathrm{~mm}$ outer diameter were used to dissipate heat from the TEG cold side. The heat pipes have a fibre wick structure with heat flux of $50 \mathrm{~W}$. Consequently, $250 \mathrm{~W}$ of heat can be moved by five heat pipes that were used. The numbers of heat pipes were chosen considering the diameter of the heat pipe, the size of the thermoelectric generator and the heat carrying capacity of each heat pipe.

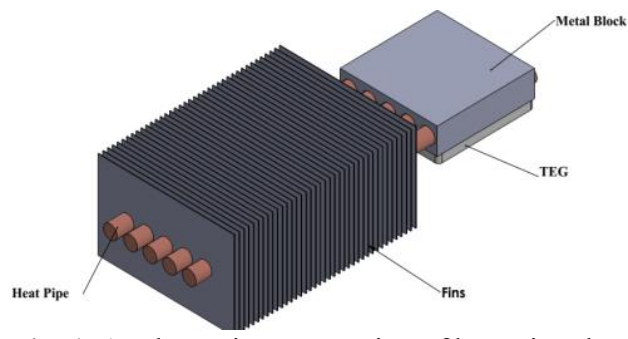

Fig. 1. A schematic presentation of heat pipe- heat sink with TEG

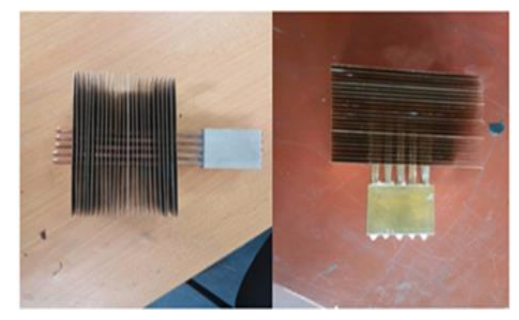

Fig. 2. Picture of aluminum and cooper heat sinks

\subsection{Testing and data collection}

Testing of the model was carried out as shown in Fig. 3. An electric heater was used as the waste heat source and a sliding rheostat was connected with TEG as electrical load. The TEG would provide maximum power output (Power $=$ Volts $\times$ Amps) when the Load resistance equals the TEG internal resistance ( $R$ of Load $=R$ of TEG), using the graph of "TEG resistance" from the manufacturer to determine the load resistance at $\mathrm{T}_{\text {hot }}$ and $\mathrm{Tc}_{\mathrm{old}}$. If the TEG internal resistance could not match the load precisely, then the load resistance were made slightly higher than the TEG resistance, rather than lower. The data collected were temperature (TEG at hot side and TEG at cold side), voltage and current. For logging of temperature, a 12-Channel Temperature data logger, Lutron BTM-4208SD was used. Thermocouples were placed at the hot side, cold side of the TEG, heat pipe condenser and on five positions of the fins. The data were recorded with intervals of 10 seconds for a period of 20 to 25 seconds, until the TEG power output reached steady state. The voltage were taken for both open and close circuits. For current, however, the circuit had to be loaded so the current could be measured. From the voltage and current measured, power (W) can be obtained by the conventional equation as shown below:

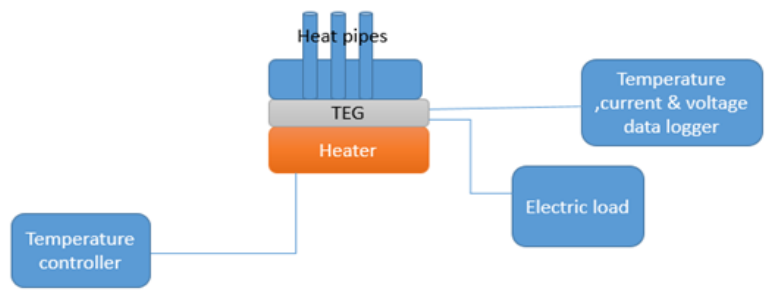

Fig. 3. Schematic of a thermoelectric generator test rig. 


$$
P=I \times V
$$

The experiments were conducted indoor to have the flexibility for a wide range of tests. Indoor experiments were conducted under simulated conditions of average annual ambient wind speed in Kuala Pahang, Malaysia. The wind speed was varied between $1.1 \mathrm{~m} / \mathrm{s}$ and 3 $\mathrm{m} / \mathrm{s}$, obtained from data collected from previous studies at Universiti Malaysia Pahang (UMP). Therefore, the cooling method was considered as operating under natural convection, which were simulated using the wind tunnel[9].

\section{Results and discussion}

Figures 4(a) and 4(b) show the comparison of TEG output between the manufacturer's charts and experiments. The two experiments can be compared with the manufacturer's charts, because the TEG cold side temperature was below $100{ }^{\circ} \mathrm{C}$, being the highest limit available from the manufacturer's data. Both experiments in Figures 4(a) and 4(b) were conducted using copper heat sink (fins of $118 \times 70 \times 0.5 \mathrm{~mm}$ ) with $3 \mathrm{~mm}$ fin space, at $300^{\circ} \mathrm{C}$ and 250 ${ }^{\circ} \mathrm{C}$ hot side temperature and $100{ }^{\circ} \mathrm{C}$ and $90{ }^{\circ} \mathrm{C}$ cold side temperature, respectively. The comparison included experimental TEG current $\left(\mathrm{C}_{\text {expr }}\right)$ with the manufacturer's TEG current $\left(\mathrm{C}_{\text {manuf }}\right)$, experimental TEG voltage $\left(\mathrm{V}_{\text {expr }}\right)$ with the manufacturer's TEG voltage $\left(\mathrm{V}_{\text {manuf }}\right)$, and the experimental power output $\left(\mathrm{P}_{\text {expr }}\right)$ with the manufacturer's power output $\left(\mathrm{P}_{\text {manuf }}\right)$, with the last parameter calculated using Equation (1) as given above. The bar charts figure 4(a) show that there is a difference of about $18 \%$ between the experimental and manufacturer's result for current, and $12 \%$ for voltage, taking the average between the respective values for current and voltage.

However, the average difference for between experimental power output and manufacturer's power output after multiplication of the respective current and voltage is only $7.9 \%$, whilst in Figure 4(b) was 9.4. The power output difference between experimental result and manufacturer's result is $0.8 \mathrm{~W}$ in Figure $4(\mathrm{a})$ and $0.5 \mathrm{~W}$ in Figure $4(\mathrm{~b})$. With less than $10 \%$ difference in power output between experimental result and manufacturer's result, the experiments can be considered as fairly accurate and acceptable, and further experiments can therefore be pursued. Figures 5 and 6 show experimental results for current, voltage and power for natural convection air flow, using copper and aluminium fins, respectively. The TEG setup was placed in a wind-tunnel to simulate the average of outdoor air speed throughout the year. The air speed inside the wind-tunnel was set and maintained at $1.7 \mathrm{~m} / \mathrm{s}$ \pm 0.2 and the temperature of the surrounding was $29^{\circ} \mathrm{C}$. The TEG hot side temperature was $300{ }^{\circ} \mathrm{C}$.

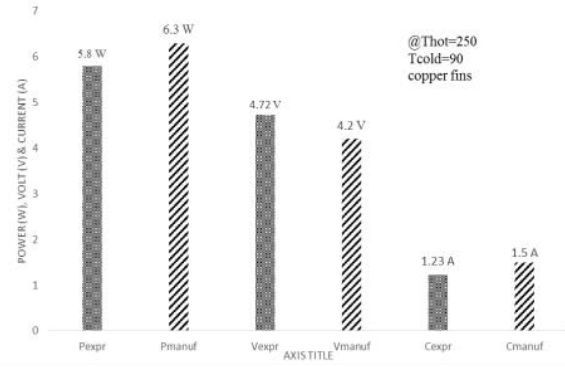

(a)

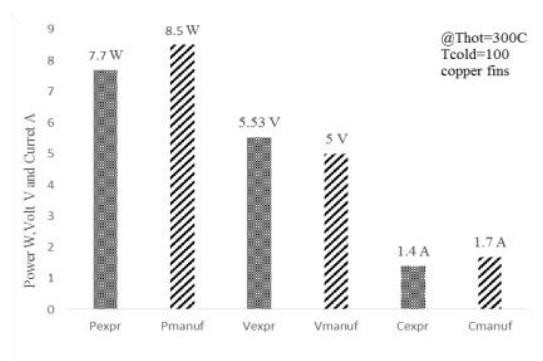

(b)

Fig. 4. Comparison TEG output between manufacturer's result and experiments at two different temperatures using copper fins: (a) $\mathrm{T}_{\text {hot }}=300^{\circ} \mathrm{C}, \mathrm{T}_{\text {cold }}=100^{\circ} \mathrm{C}$, (b) $\mathrm{T}_{\text {hot }}=250^{\circ} \mathrm{C}$, $\mathrm{T}_{\text {cold }}=90^{\circ} \mathrm{C}$ 
Heat pipe heat sink with copper and aluminium fins used were of dimensions $118 \mathrm{~mm}$ length, $70 \mathrm{~mm}$ width and 0.5 thickness and with fins spacing of $3 \mathrm{~mm}$. The power was not measured, rather calculated as a product of current and voltage. As shown in both diagrams current, voltage and power show similar trend with values starting with a direct increase for the first 6.67 minutes then reaching steady state with readings taken for a total of 15 minutes. The values after steady state were 1.22A, $4.85 \mathrm{~V}$, and $5.9 \mathrm{~W}$ (Figure 5, with copper fins), and 0.68A, 3.9 V, and 2.67 W (Figure 6, aluminum fins).

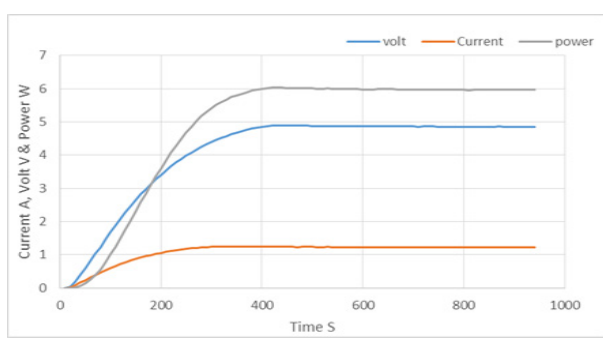

Fig. 5. Current, voltage and power vs time using copper fins (at natural convection).

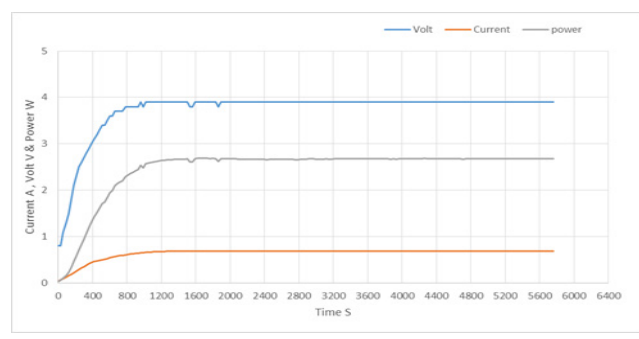

Fig. 6. Current, voltage and power vs time using aluminum fins (at natural convection).

Figures 7 and 8 show similar experiments like the above, however, under a forced convection. The forced convection using a fan with air speed of $4.2 \mathrm{~m} / \mathrm{s}$ and ambient temperature of $28^{\circ} \mathrm{C}$. The TEG hot side temperature was $300^{\circ} \mathrm{C}$. The heat pipe heat sink with copper and aluminum fins were of similar dimensions and spacing as the two experiments above. As shown in the two figures, current, voltage and power show similar trend - the steady state for current, voltage (and power) however, was reached earlier (3.33 minutes), twice faster than that of free convection. At steady state the respective values achieved were 1.4 A, 5.53 V, and 7.76 W in Figure 7 (using copper fins) and 0.76A, $4.4 \mathrm{~V}$, and $3.36 \mathrm{~W}$ in Figure 6 (aluminum fins).

It was found that the outcomes of the experiments and the manufacturer's data are wellmatched. The experiments above, in a way, verify the precision of the tests. The highest power output achieved was with the use of copper heat sink in forced convection environment, at $7.7 \mathrm{~W}$ whereas, the lowest power output achieved was with the use of aluminum heat sink in natural convection environment, at $2.67 \mathrm{~W}$. It is clear copper heat sink is better than aluminum heat sink in terms of power output. This did not take into account the energy consumed from the fan when used in the case of forced convection and the price of materials. Using self-cooling with TEG will reduce the power cost per watts $(\$ /$ w) by operate the fan from TEG itself.

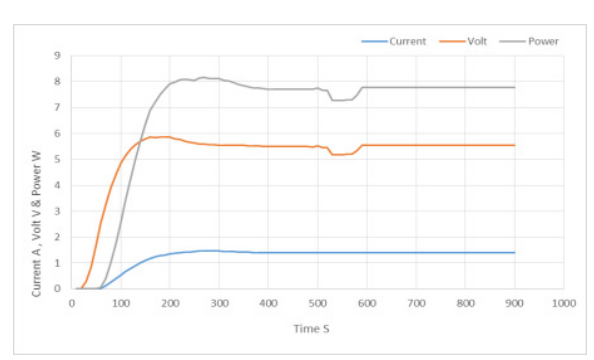

Fig. 7. Current, Voltage and Power vs time using copper fins (at forced convection)

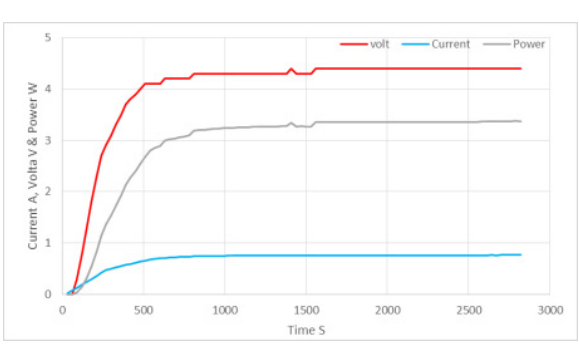

Fig. 8. Current, Voltage and Power vs time using aluminium fins (at forced convection) 


\section{Conclusion}

Two types of heat pipe-heat sink have been tested with TEG in case of natural and forced convection environment. One is made of copper and the other is aluminium (A1100). Initially, a comparison was made between experimental results and the manufacturer's data within the available range of TEG cold side temperature. When the comparison proved favorably, within less than 10\% accuracy, further tests were conducted changing from copper to aluminium fins, and varying from free convection to forced convection. The highest power output achieved was when using copper heat sink and in case of forced convection, being 7.7 $\mathrm{W}$ whereas, the lowest power output was using aluminum heat sink in the case of natural convection, being $2.67 \mathrm{~W}$. This is obvious since the conductivity of copper heat sink is superior to that of aluminum heat sink as far as power yield - and this experiment is able to quantify this superiority. However, both types of heat sink needs further optimization in terms of economic cost and efficiency because the results in this paper were just in terms of power output.

The authors gratefully acknowledge Universiti Malaysia Pahang for providing the internal grant under RDU180319.

\section{References}

1. R.Ovik, Renewable and Sust. 64, 635-659 (2016).

2. K.Yazawa., Y. R. Kok, and A. Shakouri, Appl. En,. 109, 1-9 (2013).

3. W.Liu, Acta Materialia. 87, 357-376, (2015).

4. Gayner, C., P. in Materials Science. 83, 330-382, (2016).

5. A.Elghool, Enrg. Con. M., 134, 260-277, (2017).

6. A.Elghool, MATEC Web Conf. 131, (2017).

7. M.Araiz, Enrg. Con. M. 137, 155-164, (2017).

8. Lv.Song, Enrg. Con. M. 156, 167-177, (2018).

9. A. Date, A. Date, C. Dixon, R. Singh, A. Akbarzadeh. Solar Energy. 111, 201-217 (2015). 